\title{
Thin Films with Ultra-low Thermal Expansion
}

\author{
Namiko Yamamoto,* Eleftherios Gdoutos, Risaku Toda, Victor White, Harish Manohara, \\ and Chiara Daraio
}

Ultra-low coefficient of thermal expansion (CTE) is an elusive property; most conventional materials with low CTE can operate only in narrow temperature ranges and have poor mechanical properties. Low CTE materials, particularly in thin film form, are in demand for optical, ${ }^{[1,2]}$ energy, ${ }^{[3,4]}$ and microelectronic $^{[5,6]}$ applications, but their fabrication is challenging due to the poor machinability of conventional low-CTE materials. ${ }^{[7,8]}$ Here, we engineer structured free-standing thin films with ultra-low effective CTE operating in a wide temperature range, to be integrated as thermally stable space telescope mirrors. ${ }^{[2]}$ They consist of a periodic array of bi-metallic unit cells, which locally release thermal strains in a purely mechanical way. ${ }^{[9,10]}$ The thermal and optical properties of thin films were characterized experimentally. The measurements demonstrated ultra-low effective CTE $\left(-0.6 \times 10^{-6} /{ }^{\circ} \mathrm{C}\right.$ in the temperature range between room temperature and $185^{\circ} \mathrm{C}$ ) and thermally stable imaging capability. The geometry of the array can be tailored to tune the effective CTE ${ }^{[9,11]}$ on demand. These engineered thin films with tunable CTE can provide solutions to thermal fatigue and failure of light-weight devices operating in extreme thermal environments, such as space optical systems, ${ }^{[1,2]}$ MEMS/semiconductor devices including flexible electronics, ${ }^{[5,6]}$ biomedical sensors, ${ }^{[12,13]}$ and solar energy applications..$^{[3,4]}$

Conventional materials expand when heated, because the interatomic length increases with the potential energy. Materials presenting low (or negative) CTE preserve their original volume (or contract), because the interatomic length dilation upon heating is minimized due to rigidity or through transverse vibrations, or compensated with changes of ferromagnetic/ferroelectric micro-domains..$^{[7,8]}$ Examples of materials with ultra-low or negative CTE include complex metal oxides, silica glasses and their variations, and Invar (iron metal alloy). ${ }^{[7,8]}$ These materials exhibit very low absolute CTE values $\left(\sim 10^{-8}-10^{-6} /{ }^{\circ} \mathrm{C}\right)$, but have critical limitations; the oxide materials are brittle and lack structural integrity, and the metallic alloys have very narrow applicable temperature ranges.

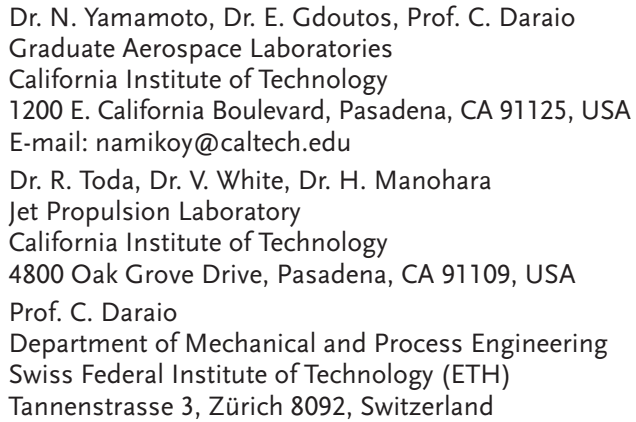

Dr. N. Yamamoto, Dr. E. Gdoutos, Prof. C. Daraio Graduate Aerospace Laboratories California Institute of Technology

1200 E. California Boulevard, Pasadena, CA 91125, USA E-mail: namikoy@caltech.edu

Dr. R. Toda, Dr. V. White, Dr. H. Manohara

let Propulsion Laboratory

California Institute of Technology

4800 Oak Grove Drive, Pasadena, CA 91109, USA

Prof. C. Daraio

Department of Mechanical and Process Engineering

Swiss Federal Institute of Technology (ETH)

Tannenstrasse 3, Zürich 8092, Switzerland

DOI: 10.1002/adma.201304997
Advanced structured materials consisting of periodic lattices of repeated unit cells with selected material properties have been shown to achieve unprecedented deformation responses governed by local deformations of the individual constituents, both theoretically ${ }^{[9,10,14-16]}$ and experimentally. ${ }^{[9-11,17-19]}$ These materials achieve low CTEs through a purely mechanical, and thus temperature-independent, mechanism. In addition, these structured materials can be designed to deliver pseudoisotropic CTE properties, unlike existing fiber-polymer composites with low/negative but anisotropic CTE properties. ${ }^{[5]}$ Macroscale structures consisting of periodic unit cells composed of two materials have been shown to present tunable CTE that is stable over a wide temperature range. ${ }^{[10,11,18]}$ However, integration of these macroscale low-CTE structures into systems, especially for the aforementioned applications, has been limited because of their inhomogeneity and rigidity. In this paper, we describe structured materials with ultra-low CTE but in self-standing thin film forms; their light-weight and integrability will allow a wider range of applications than macroscale low-CTE structures. A reliable fabrication method was developed to create and release for the first time self-standing thin films with an ultra-low CTE. Fabricated films were experimentally and analytically studied for the thermal and optical properties for application as thermally stable mirrors. ${ }^{[2]}$

The structure of the thin films consists of a two-dimensional (2D) periodic bi-material lattice (see Figure 1) composed of hexagonal plates of a higher CTE material (aluminum, $23.1 \times 10^{-6} /{ }^{\circ} \mathrm{C}$ ) combined with a frame of a lower CTE material (titanium, $8.6 \times 10^{-6} /{ }^{\circ} \mathrm{C}$ ) ${ }^{[9]}$ When heated, the thermal expansion of the hexagonal plate is accommodated by stretching and bending of the frame into the open spaces, leaving the frame's connection nodes stationary, and resulting in the low effective CTE, as illustrated in Figure 1a. In other words, bi-material lattices need to be free to locally deform in order to achieve low effective CTE. Thus, in potential applications, the thin films should be integrated on a substrate by attaching them at selected locations, not by bonding them completely to it. Meanwhile, this local release of thermal strain functions regardless of the temperature, and thus allows wide temperature ranges of application. The effective CTE can be controlled to assume negative, zero, or positive values by selecting the lattice geometry and material combination. ${ }^{[10]}$

We designed the bi-material unit cells for our mirrors using 3D finite element simulations ${ }^{[10]}$ (see Experimental Section), and targeted a CTE value for the whole film of $1.1 \times 10^{-6} /{ }^{\circ} \mathrm{C}$, as shown in Figure 1b, with consideration of space optics application. ${ }^{[2]}$ The effective CTE and local thermal strain release within the 3D plate were parametrically studied considering the effects of geometry, constituent materials properties, outof-plane deformations, substrate effects, and effective boundary conditions. The unit cells consist of two metals, to achieve high 

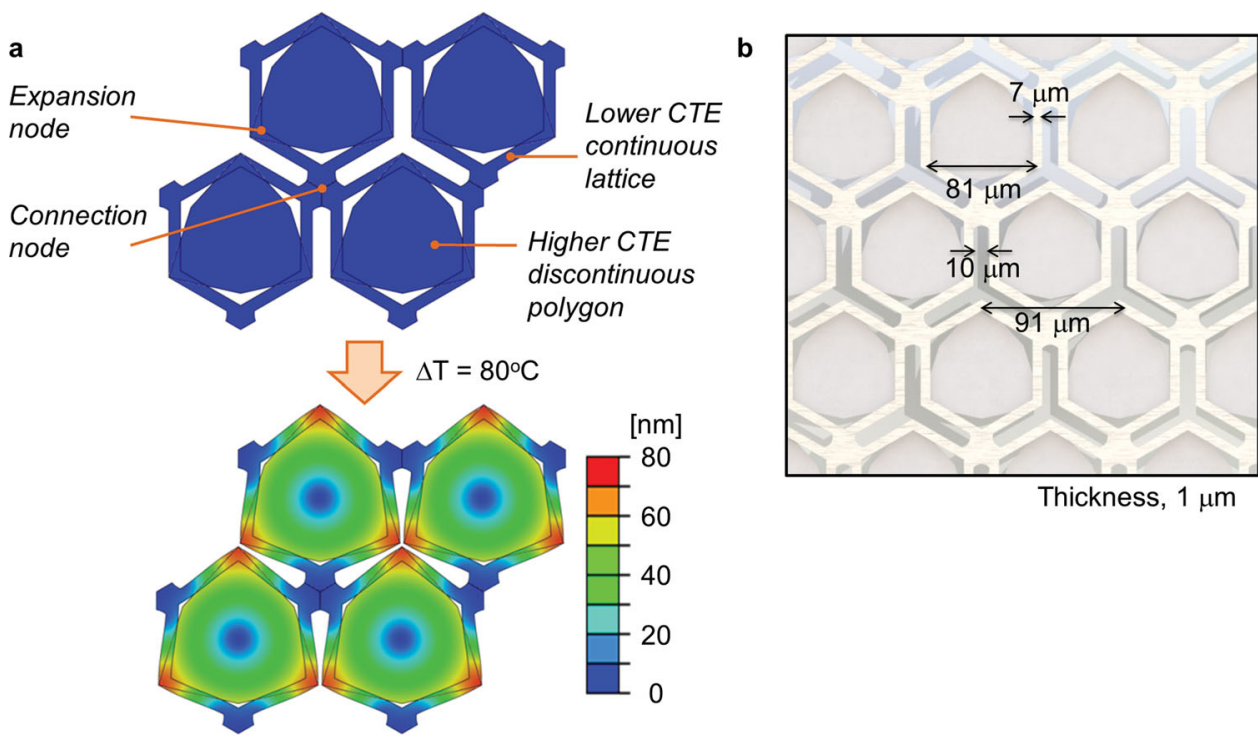

Figure 1. Design of bi-metallic lattice for tunable CTE. a, Bi-metallic lattice consists of hexagonal plates made of a higher CTE material combined with a frame of a lower CTE material. Thermal behaviors of this structure are simulated using FEM. When heated, the thermal expansion of the hexagonal plate is accommodated by stretching and bending of the frame into the open spaces. b, A bi-metallic lattice studied in this work was designed so that the aforementioned local release of thermal strains will leave the frame's connection nodes stationary, resulting in the ultra-low effective CTE.

light reflectivity and the proper CTE values: aluminum for the hexagonal plates and titanium for the frame. The frame angle and the plates' area are designed to provide a sufficient filling factor $(66 \%)$ for optical performance, and the single unit size of the periodic lattice is reduced to enhance pseudo-homogeneity. The hexagonal plates and frame are bonded by lap-joints. The structured films ( 1 $\mu \mathrm{m}$ thick) were microfabricated and

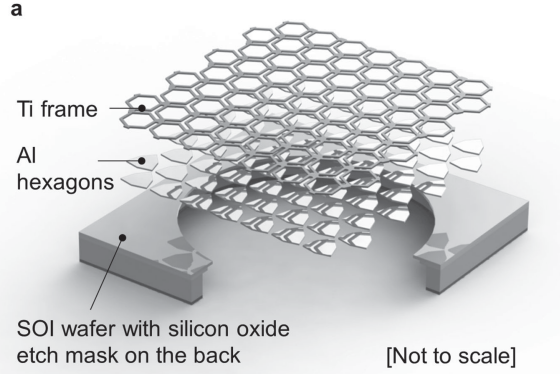

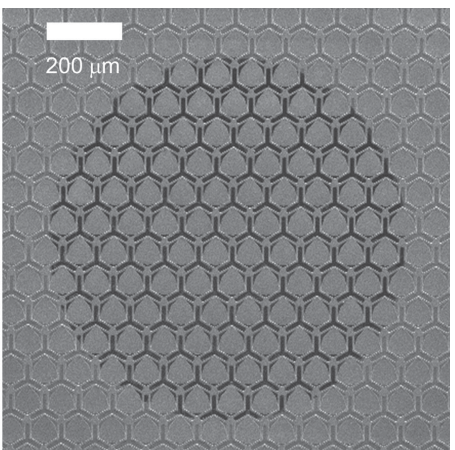

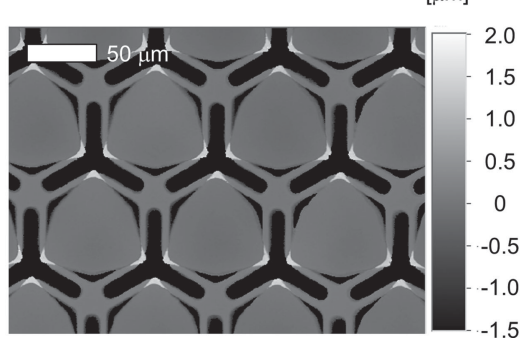

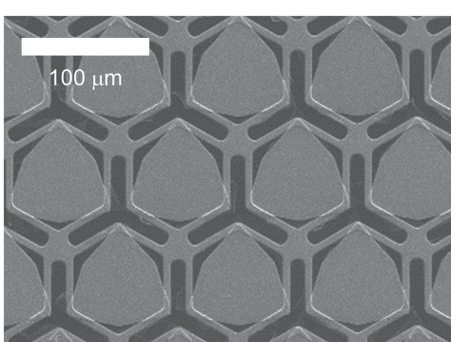

released from their substrates as $\sim 800 \mu \mathrm{m}$-diameter circular free-standing films, with a $95 \%$ yield. The fabrication details are described in Figure 2 and in Experimental Section. Optical interferometry revealed the released films to be flat, with a maximum out-of-plane variation of $\sim 0.2 \mu \mathrm{m}$ except for areas where the $\mathrm{Al}$ and Ti sections overlap (see Figure $2 \mathrm{c}$ ).

The CTE of the films was measured using a 3D digital image correlation $^{[20-23]}$ (DIC) set-up with a stereomicroscope unit (see Experimental Section and Figure S1 in Supporting Information). The measurement accuracy was evaluated using a silicon $(\mathrm{Si})$ wafer prepared with the same speckle patterns as a reference. Based on $\sim 50$ data points, the CTE of the Si wafer was calculated as $3.3 \times 10^{-6} /{ }^{\circ} \mathrm{C}$ (median), slightly larger than the literature CTE value of $\left[\begin{array}{lll}1 & 0 & 0\end{array}\right] \mathrm{Si}\left(2.6 \times 10^{-6} /{ }^{\circ} \mathrm{C}\right)$. The results of the CTE measurements are summarized in Figure 3. The mapped von Mises strains show strain concentration and thus lattice deformation around the expansion nodes, as predicted in the simulation (see Figure 3a). The films' CTE value was calculated tracking changes in the distance between points designed to be stationary: the distance between connection nodes on the frame and the distance between the centers of the hexagonal plates. The measured CTE is evaluated as $-0.6 \times 10^{-6} /{ }^{\circ} \mathrm{C}$ (median) based on 250 data points taken at locations scattered across the sample surface, at five set temperatures between room temperature and $\sim 185{ }^{\circ} \mathrm{C}$. The CTE data obtained from the structured thin films are statistically expressed in Figure $3 \mathrm{~b}$ and Figure S1b. The relatively large CTE values distribution
Figure 2. Fabrication of a bi-metallic lattice. a, A bi-metallic lattice was fabricated using the conventional micro-fabrication techniques. Discontinuous patterned films of $\mathrm{Al}$ and Ti were deposited on a substrate, and the formed lattice was released by etching the substrate from the back side. $b$, The scanning electron microscope images show the lattice released intact as a circular free-standing thin film. c, The lattice's level surface was confirmed in the optical profilometer image. 
a
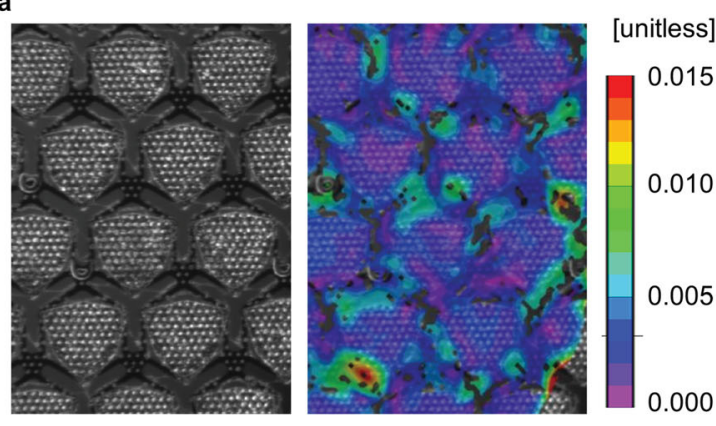

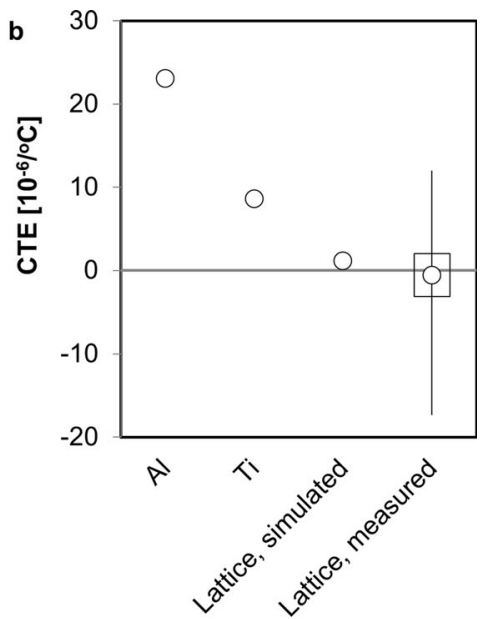

Figure 3. Measured CTEs of bi-metallic lattices, compared with simulated values. a, The stereo-microscope image (left) shows a bi-metallic lattice sample prepared with speckled patterns. In the right image, the map of von Mises strain calculated with $\Delta \mathrm{T}=116^{\circ} \mathrm{C}$ is overlaid; the large strains are observed near the rim of the hexagonal plates, as predicted by the simulation. b, Measured CTE data are compared with the CTEs of the components (Al and $\mathrm{Ti}$ ) and the simulated CTE of the bi-metallic lattice. The CTEs of bi-metallic lattice are much smaller than those of the components. The simulated and experimental CTEs are comparable. The box plot in b shows the following; circles, median; lower edge of box, $25^{\text {th }}$ percentile; upper edge of box, $75^{\text {th }}$ percentile; whiskers, extreme data points.

observed can be attributed to the optical resolution limits of the 3D DIC measurement technique, and not to intrinsic shortcomings of the structured thin film. This is evident from the fact that a similar CTE values distribution, based on the values of 25th and 75th percentiles, was observed in the measurement of bulk Si reference samples (see Figure S1b).

The measured median CTE value $\left(-0.6 \times 10^{-6} /{ }^{\circ} \mathrm{C}\right)$ is comparable with the designed CTE value $\left(1.1 \times 10^{-6} /{ }^{\circ} \mathrm{C}\right)$, and is significantly lower than those of the constituent materials, $\mathrm{Al}$ and Ti. The difference between the measured and simulated CTE values is comparable with the error range $\left(\sim 0.5 \times 10^{-6} /{ }^{\circ} \mathrm{C}\right)$ of the DIC measurement technique, as observed in the measurement of the Si reference sample (see Figure S1b). In addition, the difference can be due to the effect not modeled in the FEM simulation, such as the film residual stresses. The scattering present in the experimental data can be attributed to the out-ofplane deformation of the film during heating. For example, with $\sim 80{ }^{\circ} \mathrm{C}$ temperature increase, the out-of-plane displacement was measured using the DIC technique to be $0.1-1 \mu \mathrm{m}$, and was numerically simulated using the $3 \mathrm{D}$ FEM to be $0.2-4 \mu \mathrm{m}$. In the numerical simulation, these out-of-plane deformations resulted in spatial variation of the simulated effective CTE $\left(\sim 1.0-1.5 \times 10^{-6} /{ }^{\circ} \mathrm{C}\right)$; the CTE value decreases towards the lattice center with increasing deflection.

We evaluated the reflective properties of the low-CTE films using two methods (see Figure S2 in Supporting Information); we inspected the diffraction patterns of the lattices (see Figure 4a), and analyzed the quality of reflected images during thermal cycles. For the diffraction analysis, collimated monochromatic light (633 nm wavelength) was reflected on the films, and then focused on a CCD camera. The diffraction patterns acquired show hexagonally symmetric scattering, originated from the lattice periodicity. The reflected encircled energy was calculated by integrating the light intensity along the radial axis starting from the focus center. The encircled energy of the structured films is $-55 \%$ of that of a highly reflective continuous Al reference sample, roughly corresponding to the lattice's filling factor. Both the diffraction pattern and encircled energy match the results predicted by fast Fourier tranform assuming Fraunhofer diffraction. We also inspected the quality of images reflected on the samples during thermal cycling, and compared the results with images reflected on a reference $\mathrm{Al}$ thin film under the same heating conditions (see Figure $4 \mathrm{~b}$ ). The grid pattern reflected on a continuous Al film is clear at room temperature, but becomes defocused at an elevated temperature $\left(150^{\circ} \mathrm{C}\right)$ as the film buckles in the out-of-plane direction due to the thermal strain. Meanwhile, the reflected image quality on the structured thin films stays intact regardless of the heating. These image quality shifts are quantitatively evaluated using linear correlation coefficients between the images taken at room temperature and at $150{ }^{\circ} \mathrm{C}$ (see Experimental Section). The coefficient calculated for the structured thin films is 0.89 , while that for the continuous $\mathrm{Al}$ films is 0.53 . This result shows that the structured thin films can obtain improved image quality under thermal cycling, as compared to continuous reflective metallic layers.

In summary, the thermal and optical characterization performed in this study has demonstrated the functionality of bimetallic, structured thin films as thermally stable mirror segments. We studied the correlation between the micro-structure design and the resulting CTE properties, and described a versatile microfabrication technique to realize structured thin films on a Si substrate. The analysis and measurement of the thermomechanical responses of these thin films provided fundamental insights on the robustness of the proposed micro-lattice design in thin film form. Such knowledge obtained from this study will help extend the design regime of $2 \mathrm{D}$ and $3 \mathrm{D}$ micro-structured thin films and the systems integrated with these thin films, for future applications in micromechanical devices and energy systems. When integrated, the overall CTE properties of the systmes will be affected by the interfaces and the substrate. Tunable CTE of these thin films come advantageous as they 

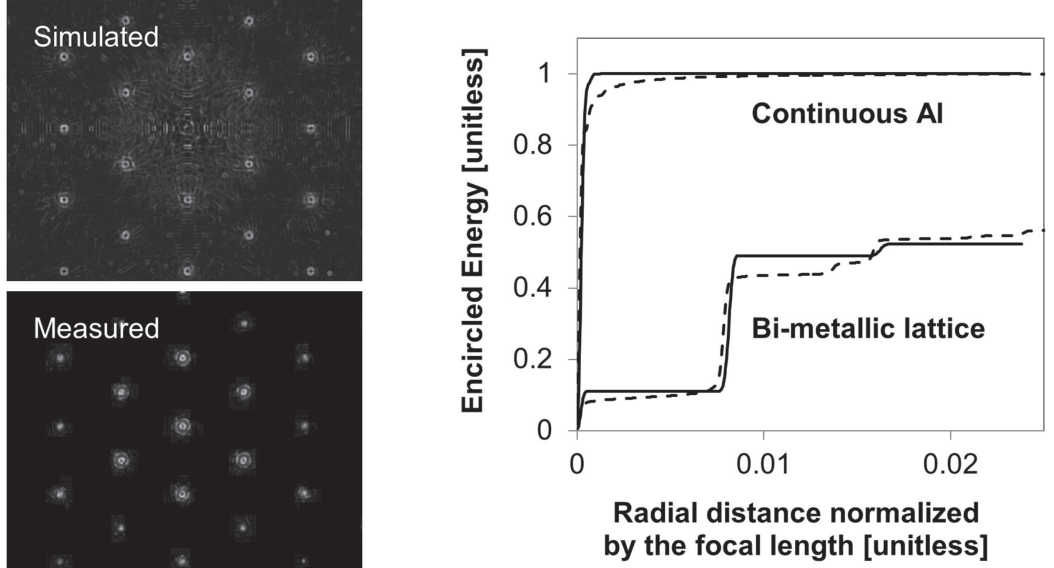

b

\section{Continuous Al}
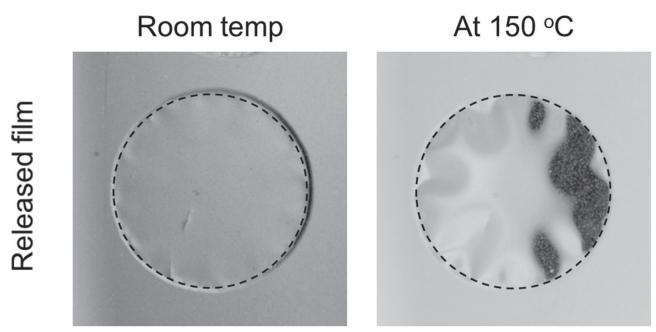

\section{Radial distance normalized by the focal length [unitless]}

\section{Bi-metallic lattice}

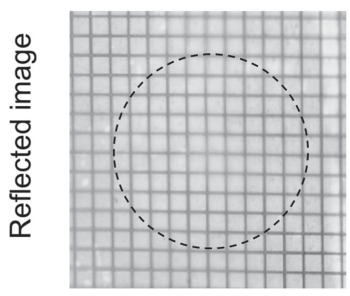

$100 \mu \mathrm{m}$

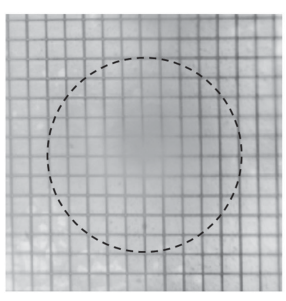

[Light intensity]
Room temp
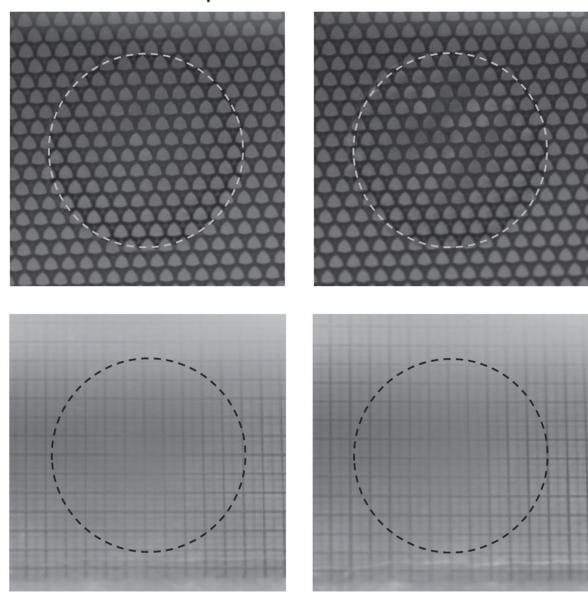

Figure 4. Demonstration of a bi-metallic lattice with ultra-low CTE as a thermally stable reflective layer. a, The light reflection of a bi-metallic lattice is evaluated through diffraction patterns. The simulated and measured diffraction patterns (left) are comparable, showing hexagonal scattering. Encircled energy (right) was calculated from the light intensity distribution of the diffraction patterns. The continuous lines show measurement, and the dotted lines show simulation. In addition to the bi-metallic lattice, a sample consisting of continuous Al film was evaluated as a perfectly reflective reference. $b$, Thermal stability of reflected images during heating is evaluated. When heated, the image reflected on a continuous Al reference film becomes defocused as the film buckles in the out-of-plane direction. Meanwhile, the reflected image quality on the bi-metallic lattice stays intact regardless of the heating. Overlaid circles on the images are showing the free-standing film areas.

can be designed (for example to be negative) to compensate for those factors to achieve the desired thermal properties.

\section{Experimental Section}

Finite Element Simulation: A full 3D model consisting of one hexagonal plate laid down on a partial frame (single unit) was simulated using 10 node tetrahedral elements. The width across the bi-metallic lattice array is $\sim 800 \mu \mathrm{m}$ with 9 hexagonal plate units repeated. The lattice rim points were fixed on a Si substrate. The interface between the hexagonal plate and the frame was modeled as bonded. Thermal displacements were computed under the load of $80^{\circ} \mathrm{C}$. The CTEs were calculated by measuring the length expansion between the thermally stable points (the frame connection nodes, or the hexagon center points) on the single unit.
Sample Fabrication: The fabrication starts with a Silicon-on-Insulator (SOI) wafer substrate. First, patterned $\mathrm{Al}$ and Ti films were deposited on a substrate using a combination of photolithography, electronbeam evaporation, and metal lift-off processes. The film thickness was measured to be $\sim 1.2 \mu \mathrm{m}$ (vs. $1 \mu \mathrm{m}$ in design), and the crystalline orientation was observed as [ [ $\left.\begin{array}{lll}1 & 1 & 1\end{array}\right]$ on the Si $\left[\begin{array}{lll}1 & 0 & 0\end{array}\right]$ substrate using X-ray diffraction. The residual stresses of both metal layers were controlled to be slightly tensile by post-deposition annealing. Second, the structured thin film was released by step-by-step etching of the substrate from the back side, using deep reaction-ion etching for the bulk $\mathrm{Si}$, reaction ion etching for the oxide layer, and then $\mathrm{XeF}_{2}$ etching for the Si device layer.

DIC Measurement: DIC is a computer-based process that provides full-field, real-time displacement measurement by tracking the motion of speckle patterns on a deforming sample. The DIC method was selected for our measurement because this technique can measure very small displacements, and because the full-field displacement map can capture the lattice deformation behavior. The structured thin films were 
prepared with a $\sim 4 \mu \mathrm{m}$ speckle patterns using photolithography. During heating from room temperature to $\sim 185{ }^{\circ} \mathrm{C}$, magnified images were recorded from two angles through a stereomicroscope, to obtain the $3 \mathrm{D}$ displacement information. Displacements were calculated by minimizing a least-squares correlation coefficient of the grayscale intensity values before and after deformation, within small neighborhoods of patterns called subsets. Interpolation process between pixels for minimization provides subpixel accuracy in the correlated displacement field. The correlation process and distortion were calibrated by measurement on reference samples of dot grids and speckle patterns. The displacement noise was evaluated by taking multiple stationary images of a sample; the noise was less than $\sim 2 \mathrm{~nm}$, in comparison with the expected displacement range of $\sim 5-15 \mathrm{~nm}$. The experimental set-up is illustrated in Figure $\mathrm{S} 1$ in Supporting Information.

Correlation Coefficient Calculation: Gray-scale bitmap pictures of the reflected images are taken as 2D arrays of light intensities. The linear (Bravais-Pearson) correlation coefficient is calculated in the following equation, where the $2 \mathrm{D}$ matrices, $A$ and $B$, are the $2 \mathrm{D}$ arrays based on the images taken at room temperature and at $150{ }^{\circ} \mathrm{C}$. This correlation coefficient can be an indicator of defocus or light intensity distribution change due to out-of-plane deformation.

$$
\text { (Correlation Coefficient })=\frac{\sum m \sum n\left(A_{m n}-\bar{A}\right)\left(B_{m n}-\bar{B}\right)}{\sqrt{\sum m \sum n}\left(A_{m n}-\bar{A}\right)^{2} \sqrt{\sum m \sum n}\left(B_{m n}-\bar{B}\right)^{2}}
$$

\section{Supporting Information}

Supporting Information is available from the Wiley Online Library or from the author.

\section{Acknowledgements}

The authors acknowledge Prof. Craig A. Steeves from University of Toronto, Mr. Keith Patterson from California Institute of Technology, Dr. Andrew A. Shapiro and Dr. James Breckinridge from Jet Propulsion Laboratory, and Ms. Elisha Byrne from the Correlated Solutions, Inc. for helpful discussions and technical assistance. This work was supported by the Keck Institute for Space Studies, Center Innovation Funds (CIF) from Jet Propulsion Laboratory. A part of this research was carried out at the Jet Propulsion Laboratory, California Institute of Technology, under a contract with the National Aeronautics and Space Administration. (C) 2010 California Institute of Technology. Government sponsorship acknowledged.
Received: October 7, 2013

Revised: January 14, 2014

Published online: February 22, 2014

[1] R. Bastaits, G. Rodrigues, J. Ph, P. Hagedorn, A. Preumont, Smart Materials and Structures 2012, 21, 064004.

[2] K. Patterson, S. Pellegrino, Appl. Opt. 2013, 52, 5327.

[3] K. Zweibel, Solar Energy Materials and Solar Cells 2000, 63, 375.

[4] K. L. Chopra, P. D. Paulson, V. Dutta, Progress in Photovoltaics: Research and Applications 2004, 12, 69.

[5] C. Zweben, JOM 1998, 50, 47.

[6] M. R. Werner, W. R. Fahrner, IEEE Transactions on Industrial Electronics 2001, 48, 249.

[7] R. Roy, D. K. Agrawal, H. A. McKinstry, Annual Review of Materials Science 1989, 19, 59.

[8] W. Miller, C. W. Smith, D. S. Mackenzie, K. E. Evans, J. Mater. Sci. 2009, 44, 5441.

[9] C. A. Steeves, S. L. dos Santos e Lucato, M. He, E. Antinucci, J. W. Hutchinson, A. G. Evans, J. Mech. Phys. Sol. 2007, 55, 1803.

[10] E. Gdoutos, A. A. Shapiro, C. Daraio, Exp. Mech. 2013, 1.

[11] C. Steeves, C. Mercer, E. Antinucci, M. He, A. Evans, International Journal of Mechanics and Materials in Design 2009, 5, 195.

[12] G. Harsanyi, Sensors in Biomedical Applications: Fundamentals, Technology and Applications, CRC Press, 2000.

[13] K. Kuribara, H. Wang, N. Uchiyama, K. Fukuda, T. Yokota, U. Zschieschang, C. Jaye, D. Fischer, H. Klauk, T. Yamamoto, K. Takimiya, M. Ikeda, H. Kuwabara, T. Sekitani, Y.-L. Loo, T. Someya, Nat. Commun. 2012, 3, 723.

[14] R. Lakes, J. Mater. Sci. Letters 1996, 15, 475.

[15] O. Sigmund, S. Torquato, Appl. Phys. Lett. 1996, 69, 3203.

[16] O. Sigmund, S. Torquato, J. Mech. Phys. Sol. 1997, 45, 1037.

[17] J. Qi, J.W. Halloran, J. Mater. Sci. 2004, 39, 4113.

[18] J. Berger, C. Mercer, R. M. McMeeking, A. G. Evans, J. Am. Cer. Soc. 2011, 94, s42.

[19] C. A. Steeves, A. G. Evans, J. Am. Cer. Soc. 2011, 94, s55.

[20] M. A. Sutton, W. J. Wolters, W. H. Peters, W. F. Ranson, S. R. McNeill, Image and Vision Computing 1983, 1, 133.

[21] T. C. Chu, W .F. Ranson, M. A. Sutton, Exp. Mech. 1985, 25, 232.

[22] M. A. Sutton, J. H. Yan, V. Tiwari, H. W. Schreier, J. J. Orteu, Optics and Lasers in Engineering 2008, 46, 746.

[23] M. A. Sutton, X. Ke, S. M. Lessner, M. Goldbach, M. Yost, F. Zhao, H. W. Schreier, J. Biomed. Mater. Res. Part A 2008, 84A, 178 